\title{
DNA methylation fingerprint of neuroblastoma reveals new biological and clinical insights
}

\begin{abstract}
Aim: To define the DNA methylation landscape of neuroblastoma and its clinicopathological impact. Materials \& methods: Microarray DNA methylation data were analyzed and associated with functional/regulatory genome annotation data, transcriptional profiles and clinicobiological parameters. Results: DNA methylation changes in neuroblastoma affect not only promoters but also intragenic and intergenic regions at cytosine-phosphate-guanine $(\mathrm{CpG})$ and non-CpG sites, and target functional chromatin domains of development and cancer-related genes such as CCND1. Tumors with diverse clinical risk showed differences affecting $\mathrm{CpG}$ and, remarkably, non-CpG sites. Non-CpG methylation observed essentially in clinically favorable cases was associated with the differentiation status of neuroblastoma and expression of key genes such as ALK. Conclusion: This epigenetic fingerprint of neuroblastoma provides new insights into the pathogenesis and clinical behavior of this pediatric tumor.
\end{abstract}

Keywords: $A L K \bullet C C N D 1 \bullet$ development $\bullet$ DNA methylome $\bullet$ neuroblastoma $\bullet$ non-CpG sites $\bullet$ nonpromoter methylation

Neuroblastoma, the most frequently occurring solid tumor of childhood, originates from embryonal precursor cells committed to the development of the sympathetic nervous system [1,2]. The clinical behavior of this pediatric malignancy is heterogeneous, whereby spontaneous regression or maturation occurs generally in infants, and aggressive progression is frequently observed in older children with disseminated disease [1-3]. The complex and heterogeneous clinical evolution of neuroblastoma is strongly associated with the patient's age at diagnosis, the extent of the disease as well as with histology and genetic features of the tumor [1]. Specific recurrent chromosomal aberrations, the amplification status of the MYCN oncogene as well as DNA ploidy alterations are generally predictive of neuroblastoma behavior; however, these alterations do not fully define the causes of this aggressive pediatric tumor [4-7].

Recent evidence has shown that pediatric solid tumors, including neuroblastoma, har- bor a paucity of recurrent genetic mutations as compared with other tumors from adults, suggesting that additional mechanisms such as epigenetic alterations may play an important role in the molecular pathogenesis of these developmental tumors [4-7].

DNA methylation of cytosines in the context of $\mathrm{CpG}$ dinucleotides is a major regulatory mechanism implicated in cellular processes that are critical for mammalian development [8]. During development, the DNA methylome undergoes genome-wide epigenetic modifications that determine developmental decisions and define the cellular identity [8]. This process sets the basis of cell-specific DNA methylation patterns that persist throughout the lifetime of postmitotic cells [8]. Genomewide studies have reported extensive disruption of $\mathrm{CpG}$ methylation patterns in cancer cells, as a result of global hypomethylation and local hypermethylation [9].

However, cytosine methylation also occurs in sites other than CPG dinucleo-
Soledad Gómez: ${ }^{\ddagger}$, Giancarlo Castellano ${ }^{\ddagger 2}$, , Gemma Mayol ${ }^{\ddagger}, 1$, Mariona Suñol ${ }^{3}$, Ana Queiros ${ }^{2}$, Marina Bibikova ${ }^{4}$, Kristopher L Nazor ${ }^{5}$, Jeanne F Loring ${ }^{5}$, Isadora Lemos', Eva Rodríguez', Carmen de Torres', Jaume Mora', José I Martín-Subero ${ }^{2,6}$ \& Cinzia Lavarino ${ }^{*, 1}$

'Developmental Tumor Biology Laboratory, Hospital Sant Joan de Déu, Edificio Docente 4th floor, C/ Santa Rosa 39-57, 08950 Esplugues de Llobregat,

Barcelona, Spain

${ }^{2}$ Institut d'Investigacions Biomèdiques August Pi i Sunyer (IDIBAPS), Barcelona, 08036, Spain

${ }^{3}$ Department of Pathology, Hospital Sant Joan de Déu, Barcelona, 08950, Spain ${ }^{4}$ Illumina, Inc., San Diego, CA 92122, USA

${ }^{5}$ Center for Regenerative Medicine, Department of Chemical Physiology, The Scripps Research Institute, La Jolla, CA 92037, USA

${ }^{6}$ Department of Anatomic Pathology, Pharmacology \& Microbiology, University of Barcelona, Barcelona, 08036, Spain *Author for correspondence:

Tel.: +34932804000

Fax: +34936009751

clavarino@fsjd.org

${ }^{\ddagger}$ Authors contributed equally 
tides ( $\mathrm{mCHG}$ and $\mathrm{mCHH}$, where $\mathrm{H}=\mathrm{A}, \mathrm{C}$ or $\mathrm{T}$ ) [10]. It has recently been described that the methylome of embryonic stem cells (ESCs), adult human and mouse brain cortex are enriched for methylation at non$\mathrm{CpG}$ sites [11-15]. The biological importance of this epigenetic phenomenon is still unknown. Similar to $\mathrm{CpG}$ methylation, non- $\mathrm{CpG}$ methylation undergoes a widespread reconfiguration during brain development from fetal to young adult [11]. Interestingly, non$\mathrm{CpG}$ methylation levels are negligible in fetal cortex, suggesting that the non-CpG methylation detected in ESCs is largely lost upon cell differentiation and then restored in neurons at later developmental stages [11]. However, DNA methylation at non-CpG contexts has rarely been described in cancer cells [16-19].

The great majority of studies that have investigated DNA methylation in neuroblastoma covered only a small fraction of the methylome of this tumor, specifically $\mathrm{CpG}$ islands (CGIs) and promoter regions, and were mainly focused on the analysis of specific genes of potential prognostic relevance [20-29]. Such studies rarely provide insights into DNA methylation changes outside these canonical sites. In our study, we have analyzed the DNA methylome of neuroblastoma, an embryonal tumor, using high-density microarrays with the aim of detecting epigenetic modifications at a genome-wide level and identifying clinicobiological associations that shed light into the molecular pathogenesis of this tumor and, potentially of other developmental cancers.

\section{Materials \& methods}

Patients, samples \& cell lines

In total, 35 primary neuroblastoma tumors (including six stage 1 , nine stage 3 , six stage $4 s$ and 14 stage 4 ) and seven high-risk neuroblastoma cases with available paired diagnostic and postchemotherapy specimens were obtained from the Hospital Sant Joan de Déu (Barcelona, Spain). Normal fetal brain $(\mathrm{n}=2)$ and adrenal gland $(\mathrm{n}=2)$ tissues as well as ganglioneuroma samples $(\mathrm{n}=2)$ were used as reference samples. Neuroblastoma risk assessment was defined by the International Neuroblastoma Staging System (INSS) [30]. Patients' clinical and biological characteristics are displayed in Supplementary Table 1 (online at http://www.futuremedicine.com/doi/full/10.2217/ epi.15.49). This study was approved by the Institutional Review Boards. Informed consent was obtained.

Neuroblastoma cell lines SK-N-AS and IMR5 were grown under standard culture conditions.

Real-time quantitative PCR, copy number variation analysis \& DNA sequencing

See Supplementary material \& methods and references [31-33].

\section{DNA methylation microarrays}

Genomic DNA bisulfite conversion and hybridization to Infinium Human Methylation 450k BeadChip (Illumina, Inc., CA, USA) was performed at the Human Genotyping Unit of the Spanish National Cancer Center (CEGEN-CNIO, Madrid, Spain), as described previously [34].

Data from the Infinium Human Methylation 450k BeadChip of four human embryonic stem cell (hESC) lines (hESC1-4) have been previously reported [35].

\section{Data normalization, quality control \& filtering of microarray data}

Microarray data were analyzed by Minfi package available through Bioconductor (Minfi: Analyze Illumina's $450 \mathrm{k}$ methylation arrays. $\mathrm{R}$ package version 3.0.1). To exclude technical biases, we used an optimized pipeline with several filters developed at the IDIBAPS. From the initial dataset of 485,512 sites (excluding probes detecting single nucleotide polymorphisms [SNPs]), we removed those with poor detection $p$-values $(\mathrm{p}>0.01)$ and those with sex-specific DNA methylation $(n=6926)$. The remaining sites $(n=478,169)$ were used for downstream analyses. Single cytosine methylation values ( $\beta$-values) in each sample were calculated as the ratio of the methylated signal intensity to the sum of methylated and unmethylated signals. Methylation values ranged from 0 , fully unmethylated, to 1 , fully methylated cytosine. hESCs were analyzed separately using the same pipeline. Supplementary Figure 1 shows a summary of this data processing.

\section{Detection of differentially methylated CpG \& non-CpG sites}

For each cytosine, we computed the difference between the DNA methylation level in the two groups under comparison (e.g., neuroblastoma vs reference samples or low-risk [stages 1-3 and stage 4S, MYCN nonamplified; $\mathrm{n}=19$ ] versus high-risk tumors [stage 4 or MYCN amplified tumors; $\mathrm{n}=16]$ ). Only CpGs with homogeneous methylation in fetal brain and adrenal gland controls were considered. CpGs were considered differentially methylated (DM) when: the absolute methylation difference (Delta) between mean DNA methylation values of cases and references was $>0.25$; and when the false discovery rate (FDR)-adjusted Wilcoxon rank test for independent samples was $<0.05$.

As the methylation variations affecting non-CpGs are small, non-CpGs were considered differentially methylated when Delta was more than 0.1.

Standard deviation density plots were used to determine the cut-off value for $\mathrm{CpG}$ and non-CpG methylation analyses (Supplementary Figure 2A \& B). 
Genomic annotation, transcriptional

\& epigenomic characterization of

CpG \& non-CpG sites

Microarray DNA methylation data were annotated using the UCSC Genome Browser database (hy19 version) [36]. For location relative to gene, we used the following categories: 10kb_5' (from 1501 to $10,000 \mathrm{bp}$ upstream of the transcription start site [TSS]), $1.5 \mathrm{~kb} 5^{\prime}$ (from 201 to $1500 \mathrm{bp}$ upstream of the TSS), TSS_200 (from TSS to 200 bp upstream of the TSS), 5'_UTR, Exon_1 (first exon), Exon (all the others exons), Intron_1 (first intron) Intron (all introns), 3'UTR, 1.5kb_3' (from the transcription termination site [TTS] to 1500 bp downstream TTS), $10 \mathrm{~kb} \_3$ ' (from 1501 to $10,000 \mathrm{bp}$ downstream of the TTS) and intergenic regions. For the location relative to CpG Island (CGI) we used: CGI, CGI shore (0-2 kb from CGI edge), CGI shelf (from $2001 \mathrm{bp}$ to $4 \mathrm{~kb}$ from the CGI edge) and outside the CGI categories. All $\mathrm{CpG}$ and non-CpG sites were annotated according to the chromatin and transcriptional state categories defined from fetal brain tissue profiles (E082) [37].

\section{TFBS analysis}

For transcription factor binding site analysis, we used the Transcription Factor ChIP-seq database (161 factors) from ENCODE with Factorbook Motifs track from the UCSC Genome Browser [37].

\section{Functional enrichment analysis}

We used the package topGO (topGO: enrichment analysis for Gene Ontology R package version 2.12.0), and PubMed database to identify enriched Gene Ontology biological processes and over-represented biological pathways.

\section{Bisulfite pyrosequencing}

To validate DNA methylation data, bisulfite pyrosequencing was performed as previously described [38]. Primer sequences are available in Supplementary materials \& methods.

\section{Gene expression microarrays}

Hybridization of RNA samples to Affymetrix Human Genome U219 Array Plates was performed at the Functional Genomic Unit, Institute of Biomedical Research August Pi i Sunyer (IDIBAPS Genomic Unit, Barcelona, Spain) according to Affymetrix standard protocols. Data from Affymetrix CEL File were normalized and processed by the robust multichip averaged (RMA) algorithm using R statistical software and the Affymetrix library available through Bioconductor [39].
Standard deviation density plots were used to determine the cut-off value for gene expression analyses (Supplementary Figure 2C).

\section{Correlation analysis}

For the analysis of correlation between methylation and gene expression, Spearman's correlation coefficients were calculated considering all Affymetrix probe set expression values and DNA methylation levels of cytosines across the corresponding $\pm 10 \mathrm{Kbp}$ gene length. To each correlation coefficient, an adjusted p-value was assigned using p.adjust function of the $\mathrm{R}$ software.

Infinium Human Methylation 450k BeadChip platform publicly available databases

See Supplementary materials \& methods.

\section{Survival analysis}

Overall survival (OS) and event-free survival (EFS) probabilities were estimated using Kaplan-Meier method and compared by means of the log-rank test. Data were analyzed with SPSS program (version 22.0, SPSS, Inc). Relapse, progression and death from disease were considered as events. All statistical tests were two-sided. The $\mathrm{p}$-values $\leq 0.05$ were considered statistically significant.

\section{Immunohistochemical analysis}

See Supplementary materials \& methods and [40].

\section{Western blot analysis}

See Supplementary materials \& methods and [40].

\section{Results}

\section{Epigenetic \& transcriptional fingerprint of} neuroblastoma

In order to generate a global view of the DNA methylation landscape of neuroblastoma, we investigated 35 primary neuroblastoma tumors, two ganglioneuromas, four normal tissue references (two adrenal gland and two fetal brain samples) (Supplementary Table 1) and four hESC lines (hESC1 to hESC4) [35] using the Infinium Human Methylation 450k (HM450K) BeadChip (Illumina, Inc.). After quality control analysis and data filtering, the methylation status of a total of 475,077 CpGs and 3091 non-CpGs was quantified in neuroblastoma, ganglioneuroma and normal tissue reference samples (Supplementary Figure 1). One sample (NB12) was excluded due to poor detection p-values.

Principal component analysis (PCA) and unsupervised hierarchical clustering of $\mathrm{CpGs}$ with variable methylation levels ( $\mathrm{SD} \geq 0.25 ; 11,620$ CpGs, $2.4 \%)$ revealed that the genome of neuroblastoma is 
epigenetically distinct, largely hypomethylated but also with a fraction of $\mathrm{CpGs}$ that show hypermethylation as compared with reference samples and hESCs (Figures $1 \mathrm{~A} \& \mathrm{D}$ ). Using this unsupervised approach, the majority of neuroblastoma samples clustered according to established clinicobiological parameters with prognostic value, underscoring that DNA methylation patterns are associated with distinct subtypes of the disease.

A differential methylation analysis between tumor and normal reference samples (adrenal gland and fetal brain) revealed 45,891 differentially methylated (FDR $<0.05, \delta$ - $\beta$-value with absolute difference of $>0.25)$ CpG sites (41,124 hypomethylated (89.6\%) and 4767 hypermethylated CpGs [10.4\%]), involving 10,881 genes (Figure 2 \& Supplementary Table 2). Ganglioneuroma samples showed similar methylation levels to normal references in $93.2 \%$ of these CpGs (less than $0.25 \beta$-value difference) thus underscoring the neuroblastoma-associated differential methylation (Figure 2A).

Further analyses showed that neuroblastomaassociated methylation changes affected all chromosomes, with hypomethylated regions being enriched in chromosome $6(\mathrm{p}<0.05)$, whereas hypermethylated regions were more abundant in chromosomes 1 and 5 (Figure 2B).

DNA methylation changes in neuroblastoma not only affected promoters but also intragenic and intergenic regions (Figure 2C). Hypomethylation occurred mostly outside CpG islands (CGIs) and CGI shelves, whereas, hypermethylation was enriched in CGIs $(\mathrm{p}<0.001)$ (Figure 2D). Moreover, hypomethylation was significantly associated $(\mathrm{p}<0.001)$ with quiescent and polycomb repressed domains (Figure 2E). Hypermethylated CpGs were preferentially associated with genomic regions showing chromatin marks typical of bivalent chromatin domains, enhancers, promoters as well as polycomb repressed regions ( $\mathrm{p}<0.001)$ (Figure 2E).

In contrast to previous reports in cancer $[17,19,41]$, we detected significant cytosine methylation changes in non- $\mathrm{CpG}$ context ( $\mathrm{mCHG}$ and $\mathrm{mCHH}$, where $\mathrm{H}=\mathrm{A}, \mathrm{C}$ or $\mathrm{T}$ ) in neuroblastoma (Figure $1 \mathrm{~B} \& \mathrm{E}$ ). Consistent with recent reports in $\mathrm{H} 1$ and $\mathrm{H} 9$ stem cells, our hESC samples showed extensive non-CpG methylation levels [12] (Figure 1B \& E). Although in neuroblastoma tumors, non-CpG methylation was not as extensive as in hESCs, we observed 14\% (437 of 3091) of non-CpGs included in the HM450K array with variable methylation levels $(S D \geq 0.05)$ (Supplementary Figure 3A; Figure 1E). Interestingly, non-CpG methylation was mostly associated with tumors characterized by favorable clinicobiological features (patients $<18$ months of age with localized
MYCN nonamplified tumors). In contrast, nonCpG methylation in clinically unfavorable tumors (patients $>18$ months of age, stage 4 or $M Y C N$ amplified tumors) was generally very low or absent (Figure 1E). Among reference samples, adrenal gland showed the highest non-CpG methylation levels, similar to a portion of tumors with favorable characteristics, being, however, lower than the mean of low-risk neuroblastomas. On the other hand, fetal cortex and ganglioneuroma samples showed lower methylation levels, similar to high-risk neuroblastomas (Figure 1B \& E). Bisulfite pyrosequencing analysis of differentially methylated non-CpG sites performed using a set of samples included in the microarray, showed an excellent degree of correlation $\left(r^{2}=0.9 ; p<0.001\right)$ between microarray and pyrosequencing data of tumor and normal reference samples (Supplementary Figure 4), confirming thus the validity of our microarray findings.

To further investigate this epigenetic modification, we compared non- $\mathrm{CpG}$ methylation profiles of neuroblastoma with diverse human normal and cancer samples ( $\mathrm{n}=1334$ ) using 12 publicly available datasets obtained using infinium Human Methylation 450K BeadChip platform (see Supplementary materials \& methods). We analyzed non-CpG sites showing methylation changes in neuroblastoma $(n=437)$. We observed the highest methylation levels for hESCs and mature neurons, confirming the data reported in previous articles [11-15]. Other normal tissues showed lower (glia, adult cerebellum, adrenal gland) to nearly absent (child cerebellum, cord blood, lung) non-CpG methylation, in agreement with previously published data. The mean of non-CpG methylation levels of all neuroblastoma tumors, regardless of clinical risk subgroups, showed higher non-CpG methylation levels than other tumors included in this analysis, albeit lower than normal tissues (Supplementary Figure 3B).

Similar to DNA methylation, microarray-based gene expression analysis revealed distinct expression profiles between neuroblastoma and reference samples ( $S D \geq 1$, Figure 1C). We analyzed the association between DNA methylation and gene expression levels in 19 neuroblastoma tumors with available data from both array types. We identified a significant correlation in $1341 \mathrm{CpGs}$ $(\mathrm{FDR}<0.05)$ (Figure $1 F)$, corresponding to 849 genes, and in 210 non-CpGs ( $\mathrm{p}<0.05$ ) (Figure 1G) corresponding to 203 genes (Supplementary Table 3). Genecentered density plots of cytosines with a significant correlation coefficient $(p<0.05)$ showed both positive and negative correlations in the gene body, and high levels of negative and positive correlations around the Transcription Start Site (TSS; Figure $1 \mathrm{H} \& \mathrm{I}$, refers to CpGs and non-CpGs, respectively). 
Distinct DNA methylation levels of CpG \& non-CpG regions between high \& low clinical risk neuroblastoma

We analyzed differential DNA methylation patterns between neuroblastoma clinical risk subgroups (lowrisk $[n=18]$ and high-risk tumors $[n=16])$. In the CpG context, a pairwise comparison revealed distinct DNA methylation patterns that affected 2695 CpG sites. High-risk tumors were characterized by a widespread hypermethylation compared with low-risk tumors (2477 CpGs; 91.91\%) (Supplementary Table 4). DNA methylation differences were distributed throughout the genome, with autosomal chromosomes 1, $2,12,14,15,16,19$ and 22 being significantly affected with respect to background ( $<<0.001$; Supplementary Figure 5A \& Supplementary Table 4).

A comparison among neuroblastoma, reference samples and hESC methylation patterns revealed four sets of de novo methylation changes in neuroblastoma clinical groups (Figure 3A \& Supplementary Table 4). Hypermethylation in high-risk tumors (418 CpGs from 186 genes) targeted mostly enhancer (17\%), bivalent enhancers (42\%) and polycomb repressed regions $(22 \%$; $\leq \leq 0.001$; Figure 3A \& Supplementary Figure 5C-E). GO analysis revealed a significant $(\mathrm{p}<0.05)$ enrichment for genes involved in differentiation, neuron development and biosynthetic process (i.e., SOX10, TFAP2, NEUROG3, ONECUT2, ARID5A, LHX3, FOXH1, among others) (Figure $3 \mathrm{~A} \&$ Supplementary Figure $5 \mathrm{C}-\mathrm{E}$ \& Supplementary Tables 4 \& 5). Low-risk tumors showed a limited number of $\mathrm{CpGs}$ hypermethylated de novo (11 CpGs; ten genes). All these $\mathrm{CpGs}$ were located at promoter- and enhancer-related regions of genes including LIN28B, CYTL1, ARHGAP22 and INSM1.

Instead, CpG hypomethylation identified in lowrisk tumors (1029 CpG; 453 genes) targeted genebody and intergenic regions mostly outside CGIs $(\mathrm{p}<0.05$; Supplementary Figure $5 C \& D)$. These DNA methylation changes were clearly enriched for heterochromatic/quiescent $(47 \%)$ and weak repressed polycomb $(23 \%)$ regions $(\mathrm{p}<0.05)$. On the other hand, in high-risk tumors, de novo $\mathrm{CpG}$ hypomethylation was found to be more limited ( $87 \mathrm{CpG} ; 52$ genes) and affected mostly regions of polycomb repressed (43\%), together with enhancer $(32 \%)$ regions $(p<0.0001)$ (Supplementary Figure 5C-E). In general, hypomethylation in low-risk tumors significantly $(\mathrm{p}<0.05)$ affected genes related to development (i.e., FOXP1, $S O X 13, R A R R E S 3)$ and in high-risk tumors genes related to regulation of gene expression and RNA processing (i.e., FUBP1, DOM $3 Z$ and $N H P 2$, and POLR3H; Supplementary Tables 4 \& 5).
Next, we explored DNA methylation at non-CpG sites of high- and low-risk neuroblastoma tumors. Interestingly, we identified 118 methylated nonCpG-associated preferentially with the clinically more favorable tumors whereas high-risk tumors showed very low or absent non-CpG methylation (Figure 3B). Although the number of non-CpG sites probed by the methylation array was small, we identified non-CpG methylation throughout the genome and more prevalent at chromosome 2 ( $\mathrm{p}<0.05)$ (Supplementary Figure $5 \mathrm{~B})$. We analyzed the sequence surrounding non-CpG methylation ( \pm 4 bp) to determine whether enrichment of particular nucleotide combinations was evident, as previously reported [12]. We observed that nonCpG methylation took place in CpApG (53\%) and CpApC trinucleotides (37\%), being only the latter enriched as compared with the background (37 vs $5 \%, \mathrm{p}<0.001$ ) (Supplementary Figure $5 \mathrm{H}$ ). Non$\mathrm{CpG}$ methylation targeted preponderantly intragenic regions, exclusively at introns (18\%), and intergenic regions $(66 \%)$ associated with heterochromatic/quiescent domains and weak transcription chromatin marks (Supplementary Figure 5F \& G). Notably, genes showing non-CpG methylation were significantly $(p<0.05)$ associated with development, cell differentiation and the Wnt receptor signaling pathway (Supplementary Table 5).

This epigenetic modification was further investigated by comparing non-CpG methylation profiles of neuroblastoma clinical risk subgroups (low- and highrisk tumors) with the DNA methylation datasets of diverse human normal and cancer samples $(n=1334)$ (see Supplementary Materials \& Methods). All nonCpG sites showing methylation changes in neuroblastoma $(\mathrm{n}=437)$ as well as non-CpGs differentially methylated between neuroblastoma clinical risk subgroups $(\mathrm{n}=118)$ were used for the analysis. The analysis using the 437 non-CpGs (data not shown) was comparable and provided equivalent results to the analysis performed with 118 non-CpGs (Figure 3C). hESCs and human adult neurons showed consistently high non-CpG methylation levels whereas minor non-CpG methylation was observed in all the tumor samples (including high-risk neuroblastoma), except for low-risk neuroblastoma. In fact, low-risk neuroblastoma showed mean methylation levels similar to normal adult tissues, specifically to adult cerebellum.

\section{DNA methylation changes at CpG \& non-CpG} sites are associated with the clinical course of neuroblastoma

Previous studies have shown that the simultaneous methylation of multiple CGIs, the CpG island 
(A)

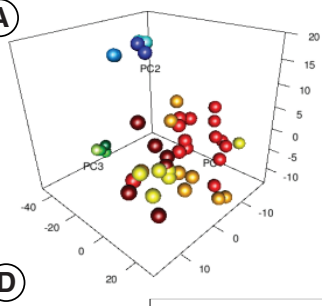

(D)

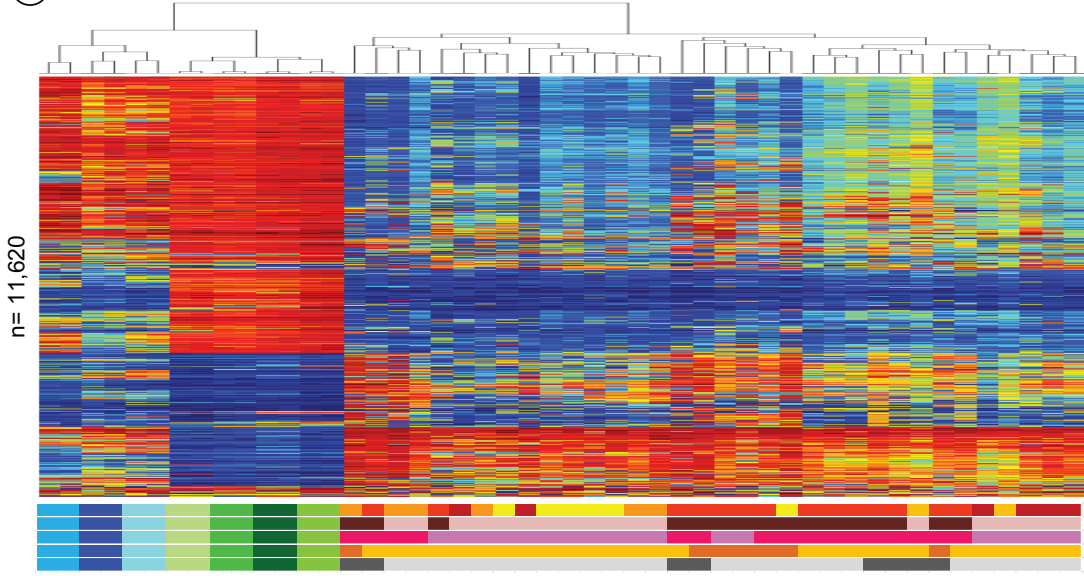

(B)

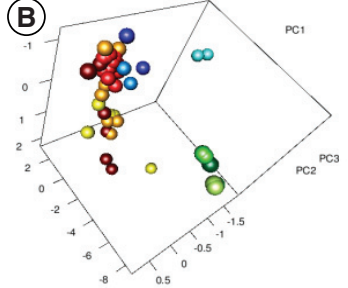

(C)

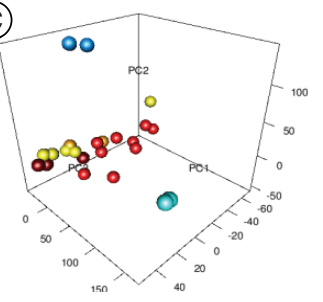

(E)

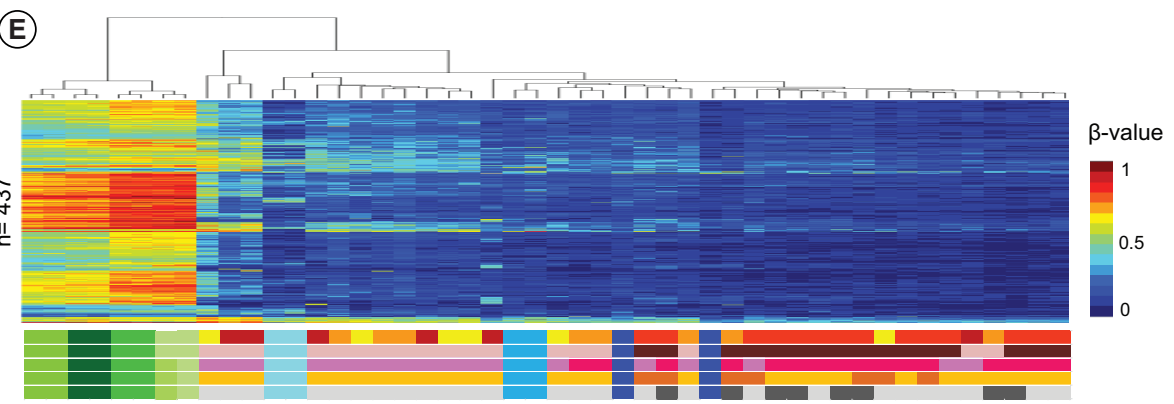

(F)

(G)

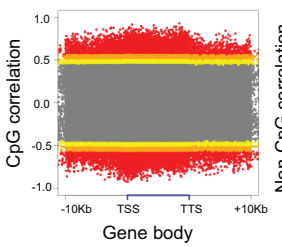

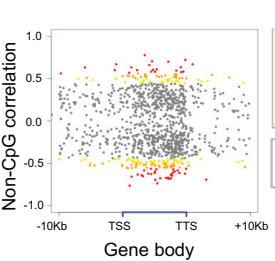

$(\mathbb{H}$

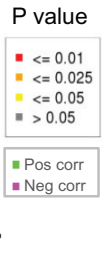

(1)

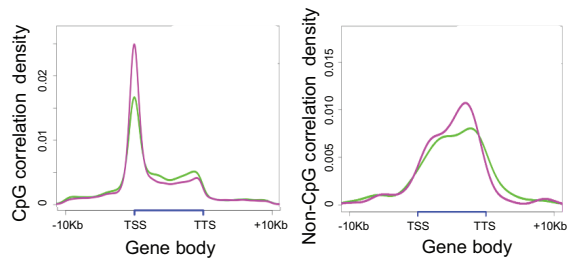
Samples

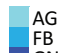

Figure 1. DNA methylation and gene expression analysis of the neuroblastoma samples included in the study. (A \& B) PCA of (A) CpG ( $S D \geq 0.25)$ and (B) non-CpG $(S D \geq 0.05)$ methylation data of neuroblastoma samples (classified according to INSS), reference samples and hESC cells. (C) PCA of gene expression data of neuroblastoma and reference samples $(S D \geq 1)$. ( $D \& E)$ Hierarchical cluster of $(D) C p G(S D \geq 0.25)$ and $(E)$ non-CpG $(S D \geq 0.05)$ methylation levels in neuroblastoma, reference samples and hESC cells. (F \& G) Correlation coefficients of gene expression and DNA methylation of single (F) CpGs and (G) non-CpGs relative to the gene-body length and $10 \mathrm{~kb}$ upstream and downstream regions. (H \& I) Normalized density plots of significant correlation coefficients $(p \leq 0.05)$ from data of panels $\mathrm{H}$ and $\mathrm{I}$, respectively.

$<18 \mathrm{~m} />18 \mathrm{~m}$ : Patients younger/older than 18 months at diagnosis; AG: Adrenal gland; Alive/dead: Survival patient status; ALL: Acute lymphoblastic leukemia; $\beta$-value: DNA methylation level (value); CLL: Chronic lymphocytic leukemia; FB: Fetal brain; GN: Ganglioneuroma; hESC: Human embryonic stem cell; HR: High-risk neuroblastoma; LR: Low-risk neuroblastoma; NA/A: MYCN status nonamplified/amplified; PCA: Principal component analysis; Pos/ Neg corr: Positive/negative correlation between gene expression and DNA methylation; St: Stage according to the International Neuroblastoma Staging System. 
methylator phenotype (CIMP), is associated with poor prognosis in neuroblastoma [21-23]. However, in neuroblastoma the CIMP is exemplified by the methylation state of the $P C D H B$ gene family (32-34). Our study confirms that high-risk neuroblastomas have more CpG methylated sites (91\%), a much higher extent of CGI methylation (93\%) and more genes (90\%) with significant CpG methylation than low-risk tumors. However, our genome-wide analysis showed that high-risk-associated methylation is extensive and targets CGIs at promoter and genebody regions of a large number of genes $(n=950)$, but also affects cytosines at functional regions (promoter and enhancer regions) outside CGIs and genes (Supplementary Table 4).
In order to identify DNA methylation changes associated with the clinical course of neuroblastoma, we selected $\mathrm{CpG}$ sites that exhibited increased methylation $(>0.75)$ in most high-risk $(>80 \%)$ but not in low-risk tumors, and analyzed their association with neuroblastoma patient outcome. Seven CpGs fulfilled our criteria, five of these were associated with genes (ANXA11, MAT1A, PIK3AP1, RLBP1 and SNED1) most of them (6/7) were located outside CGIs (Supplementary Table 6). Survival analysis showed that all seven $\mathrm{CpGs}$ were significantly associated with event-free (EFS; $\mathrm{p}<0.001)$ and overall survival (OS; p < 0.005) (Figure 4, Supplementary Table 6). We performed similar survival analysis using clinical and biological parameters (INSS, MYCN status

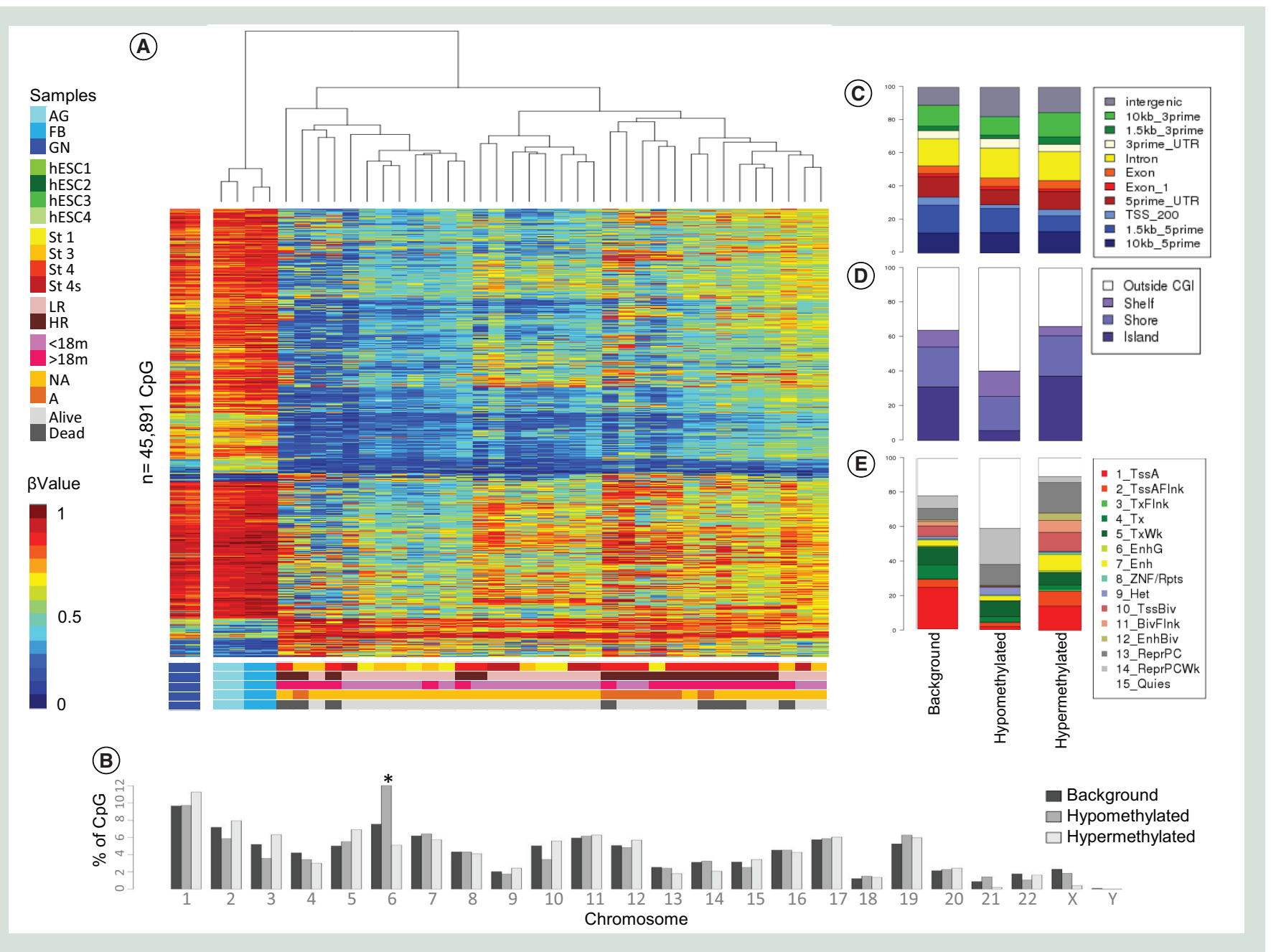

Figure 2. Differential DNA methylation between neuroblastoma and normal reference tissues. (A) Heatmap of hierarchical cluster analysis of $\mathrm{CpG}$ sites in NB with respect to reference samples. (A-left) Comparison of identified differentially methylated CpGs between NB and reference samples with GN methylation levels (B) Chromosomal distribution of differentially methylated CpG sites in neuroblastoma with respect to reference samples; $\left(^{*}\right)=p<0.05$, (C-E) Distribution of differentially methylated sites in (C) gene-related regions, (D) CpG island regions and (E) chromatin states. See Materials \& methods section for genomic annotation, transcriptional and epigenomic characterization of $\mathrm{CpG}$ and non-CpG sites.

$<18 \mathrm{~m} />18 \mathrm{~m}$ : Patients younger/older than 18 months at diagnosis; AG: Adrenal gland; $\beta$-value: DNA methylation value; FB: Fetal brain; GN: Ganglioneuroma; HR: High-risk neuroblastoma; LR: Low-risk neuroblastoma; NA/A: MYCN status nonamplified/amplified. 
(A)

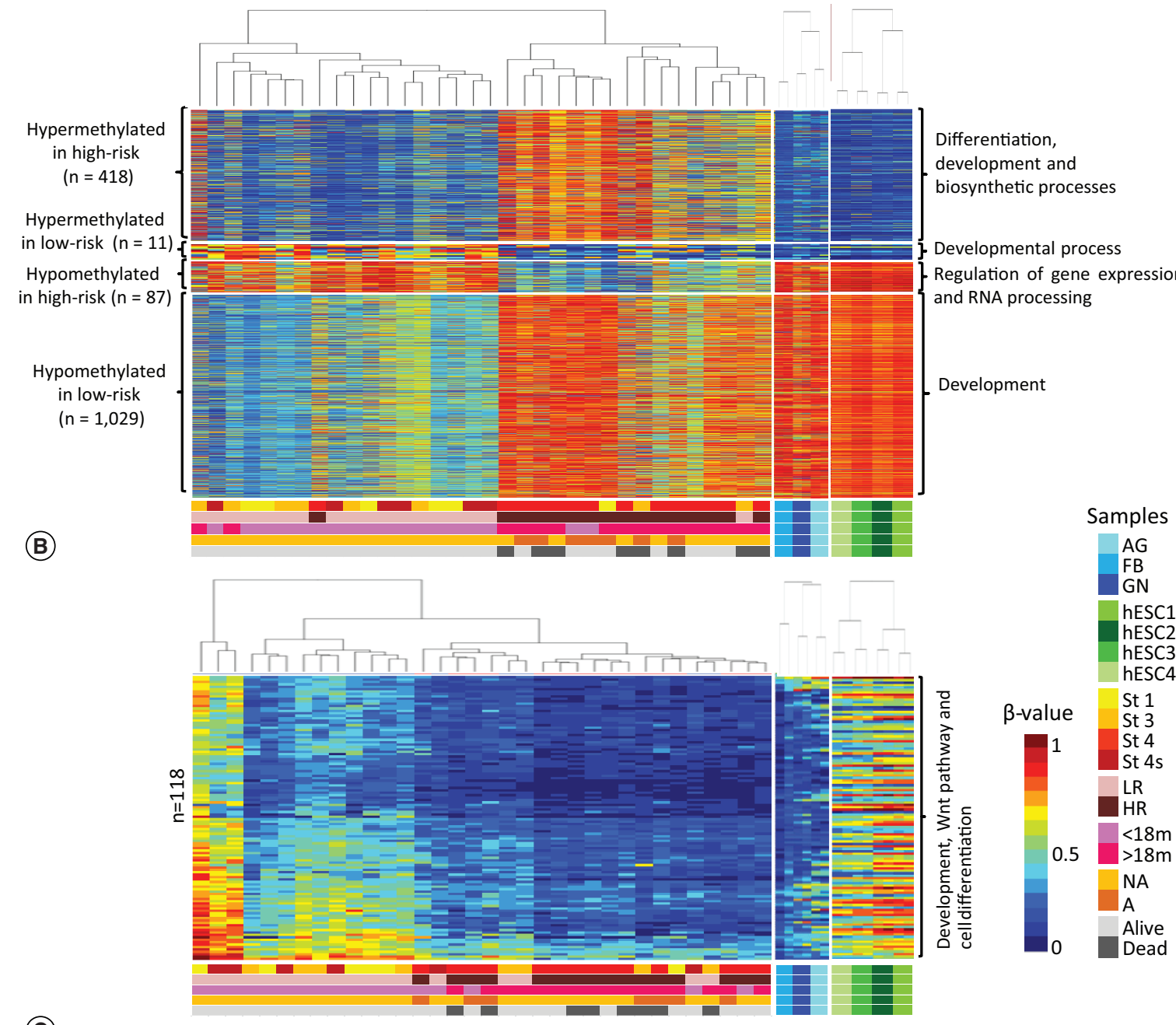

(C)

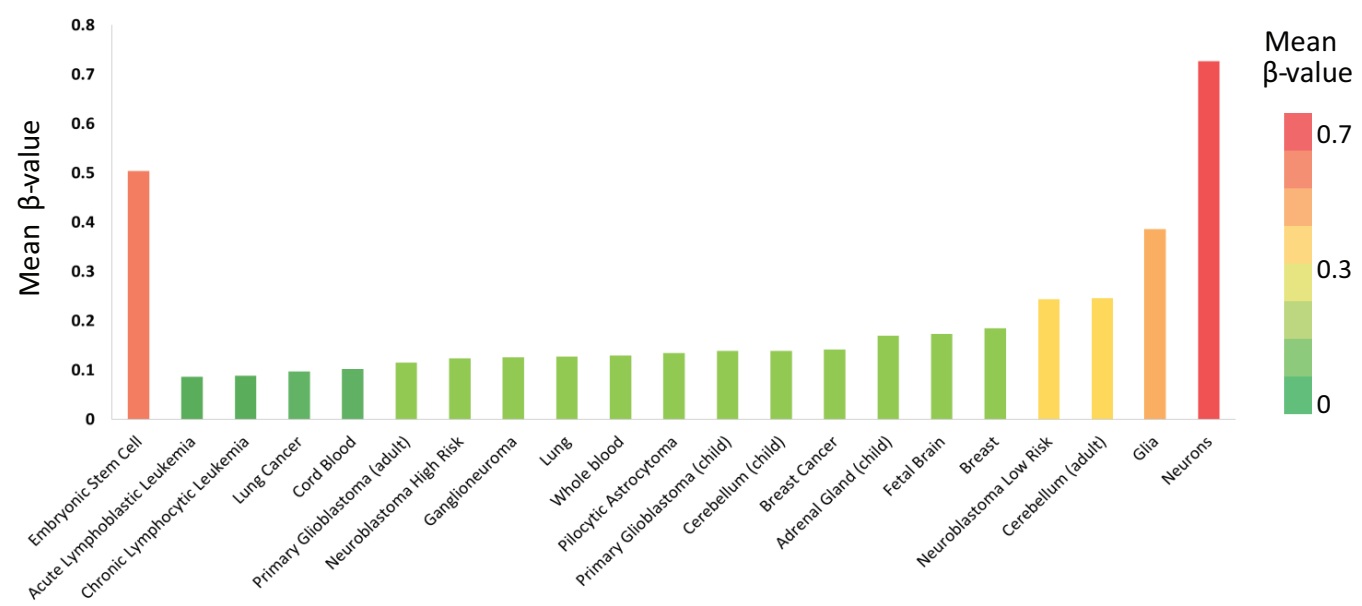

Figure 3. Distinct DNA methylation levels of $\mathrm{CpG}$ and non-CpG regions in neuroblastoma with different clinical courses. (A \& $B$ ) Heatmap of hierarchical cluster analysis of (A) CpGs and (B) non-CpGs differentially methylated in high-risk with respect to low-risk 
Figure 3. Distinct DNA methylation levels of $\mathrm{CpG}$ and non-CpG regions in neuroblastoma with different clinical courses (cont.). neuroblastoma. Comparing neuroblastoma, references samples and hESCs methylation patterns revealed four significant sets of de novo $\mathrm{CpG}$ methylation changes in neuroblastoma with different clinical behavior (panel A, left). Significantly enriched Gene Ontology terms related to genes acquiring de novo CpG and non-CpG methylation (A \& B, right). (C) Mean methylation levels of non-CpG differentially methylated in high-risk with respect to low-risk neuroblastoma bar plot from normal and cancer tissues.

$<18 \mathrm{~m} />18 \mathrm{~m}$ : Patients younger/older than 18 months at diagnosis; Alive/dead: survival patient status; AG: Adrenal gland; ALL: Acute lymphoblastic leukemia; $\beta$-value: DNA methylation level (value); CLL: Chronic lymphocytic leukemia; FB: Fetal brain; GN: Ganglioneuroma; hESC: Human embryonic stem cell; HR: High-risk neuroblastoma; LR: Low-risk neuroblastoma; NA/A: MYCN status nonamplified/amplified.

and patient's age at diagnosis) as well as differentially methylated $\mathrm{CpG}$ associated with $P C D H B$ genes and other genes with reported prognostic value in neuroblastoma [21-23]. INSS and age of patients performed similarly to CpGs identified in our study, being statistically significantly associated with both EFS ( $p<0.001$, for both) and OS ( $<<0.005$, for both; Figure 4 \& Supplementary Figure 6 ). A large proportion of the $P C D H B$ cluster showed higher methylation levels in high-risk tumors (mostly at CGI regions located at exon 1 of genes), although not reaching statistical significance in most cases. According to our cut-off-values for methylation analyses, $P C D H B 3$ and $P C D H B 7$ genes were significantly differentially methylated between tumors with diverse clinical risk (Supplementary Tables 4 \& 6). Specifically, PCDHB7 gene methylation targeting $\mathrm{CpG}$ s outside CGI region at exon 1 showed a significant $(p<0.05)$ association with overall survival in our cohort of neuroblastoma.

Next, we examined non-CpG sites that exhibited methylation (above mean non-CpG methylation levels) in most low-risk neuroblastoma (>80\%) but not in high-risk tumors. We identified ten non-CpGs, five of these were associated with genes (TLE3, ALK, PTPLAD1, CACUL1 and PRKAG2). Survival analysis showed that all these non-CpGs were significantly associated with EFS $(p \leq 0.05)$ and OS ( $\mathrm{p} \leq 0.05$ ) (Figure 4). Interestingly, non-CpGs located outside genes were more strongly associated with the clinical outcome of neuroblastoma patients (EFS $\mathrm{p} \leq 0.001$ and OS $\mathrm{p}<0.005$ ), similar to INSS, age of patients and the $\mathrm{CpG}$ sites analyzed (Figure 4 \& Supplementary Table 6).

These observations suggest that DNA methylation changes at $\mathrm{CpG}$ and non-CpG sites both inside and outside CGIs and gene-bodies are associated with neuroblastoma clinical behavior.

\section{DNA methylation variations target genes mutated or recurrently altered in neuroblastoma}

Previous reports have suggested that genetic and epigenetic alterations in cancer may affect the same genes and pathways [9,42]. We explored whether genes with recurrent genetic alterations in neuroblastoma acquired aberrant DNA methylation patterns (Supplementary Table 7) [4-7,24,43]. The gene set of interest incudes, to the best of our knowledge, genes reported to be mutated or with recurrent genetic alterations in neuroblastoma (Supplementary Table 7) [4-7,24,43]. Genes with reported differential expression but with no reported genetic alterations have not been included in the analysis. We identified differentially methylated CpGs between neuroblastoma and reference samples in a significant portion of the genes with reported genetic alterations (21 out of 28; p < 0.05; Supplementary Table 7). DNA methylation changes in these genes occurred less frequently at promoters and CGIs and targeted greatly gene-body regions (Intron 52\%; Supplementary Tables 2, 4 \& 7). All these genes showed loss of methylation in the gene body. Aberrant hypermethylation was detected in six genes and also targeted mainly gene-body CpGs. The most extensive $\mathrm{CpG}$ methylation changes were observed for CCND1, ALK and DLC1. In the case of $D L C 1$, as no correlation between DNA methylation and gene expression was observed, this gene was not further explored.

The CCND1 gene showed extensive loss of CpG methylation (23 CpGs) in gene-body and $3^{\prime}$-UTR regions in the absence of alterations in the promoter region in all neuroblastoma tumors (Figure 5A \& Supplementary Table 2). A large proportion of the differentially methylated CpGs targeting CCND1 $(12 / 23)$ showed a significant $(\mathrm{p}<0.05)$ correlation, mostly negative, with gene expression in neuroblastoma (Figure 5B). Differential expression between neuroblastoma and references samples is reported in Figure 5D. We found that these CpGs were enriched for binding sites with a high degree of affinity for the POLR2A (Supplementary Table 8). The altered methylation pattern of CCND1 was not related to the presence of gene amplification or mutation (Supplementary Table 1). Based on recent findings suggesting a close relationship between enhancer methylation upstream (CpG-TSS distance $<100 \mathrm{~kb}$ ) of CCND1 and its expression in breast cancer [44], we explored methylation of $\mathrm{CpG}$ sites in this distal region in neuroblastoma. We identified 14 differentially 
methylated (both hyper- and hypomethylated) $\mathrm{CpGs}$ located 160-190 kb upstream of the TSS of CCND1, which contained several potential enhancer regions that correlated (mostly positive correlation) with gene expression (Figure 5C). These enhancer-related CpG sites were enriched for binding sites of diverse transcription factors, among these BHLHE40, FOXA1, GATA3 and MAX, reported to have high affinity for these binding sites (Supplementary Table 8).

The ALK gene showed gene-body DNA methylation changes at both $\mathrm{CpG}$ and non-CpG sites. The promoter region was devoid of methylation both in tumors and reference samples (Figure 6A \& Supplementary Table 4). Loss of methylation at $\mathrm{CpG}$ sites was preponderant in all neuroblastoma samples and did not have a significant impact on $A L K$ expression levels. These regions were enriched for binding sites of transcription factors such as REST and CTCF (Supplementary Table 9).

Interestingly, differential methylation between high- and low-risk neuroblastoma was identified at non-CpG sites of the $A L K$ gene. These intragenic non$\mathrm{CpG}$ sites were located in introns and showed a significant inverse correlation $(\mathrm{p}<0.05)$ with gene expression (Figure 6B \& Supplementary Table 4). Notably, highrisk tumors lacked non-CpG methylation and showed higher $A L K$ expression levels, whereas, clinically favorable neuroblastomas displayed non-CpG methylation associated with lower expression levels (Figure 6C \& D). Among neuroblastoma samples, we identified one case with $A L K$ mutation (NB13: c3520T>C, p.F1174L) and one with gene amplification (NB7), both samples were high-risk tumors with unmethylated non-CpGs and high expression of $A L K$ (Supplementary Table 1).
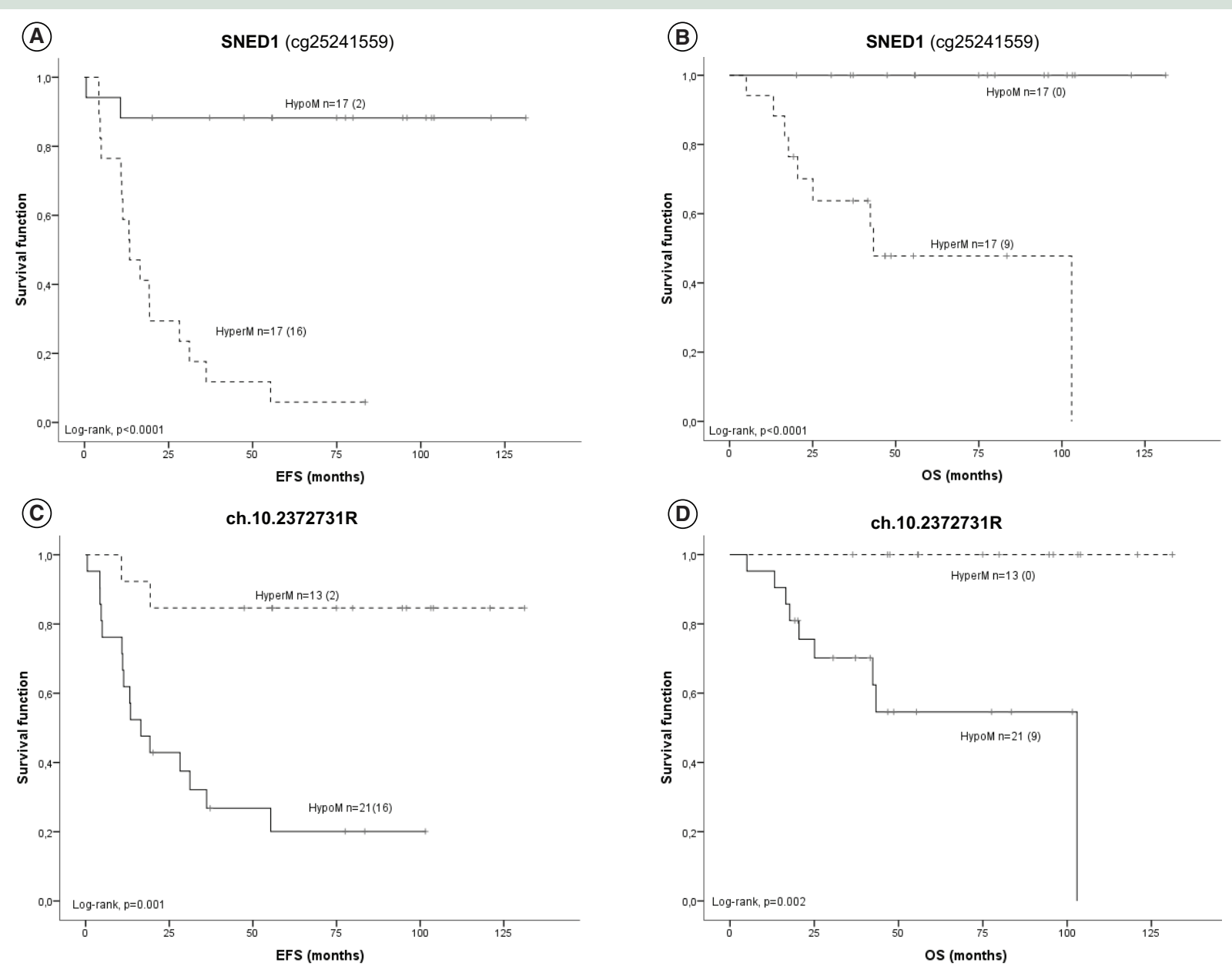

Figure 4. Kaplan-Meier survival curves and log-rank analysis. Kaplan-Meier survival curves and log-rank analysis using exemplary $\mathrm{CpG}$ and non-CpG sites found to be significantly differentially methylated between high- and low-risk neuroblastoma cases. (A \& B) CpG (cg25241559) (A) EFS HypoM $0.88 \pm 0.07$ vs HyperM $0.05 \pm 0.057 ; p<0.0001 ;$ (B) OS HypoM 1.0 vs HyperM $0.47 \pm 0.13 ; p<0.0001$. (C \& D) Non-CpG (ch.10.2372731) (C) EFS HypoM $0.20 \pm 0.09$ vs HyperM $0.84 \pm 0.10 ; p=0.001$; (D) OS HypoM $0.54 \pm 0.12$ vs HyperM 1.0 ; $\mathrm{p}=0.002$.

EFS: Event-free survival; OS: Overall survival. 
(A)
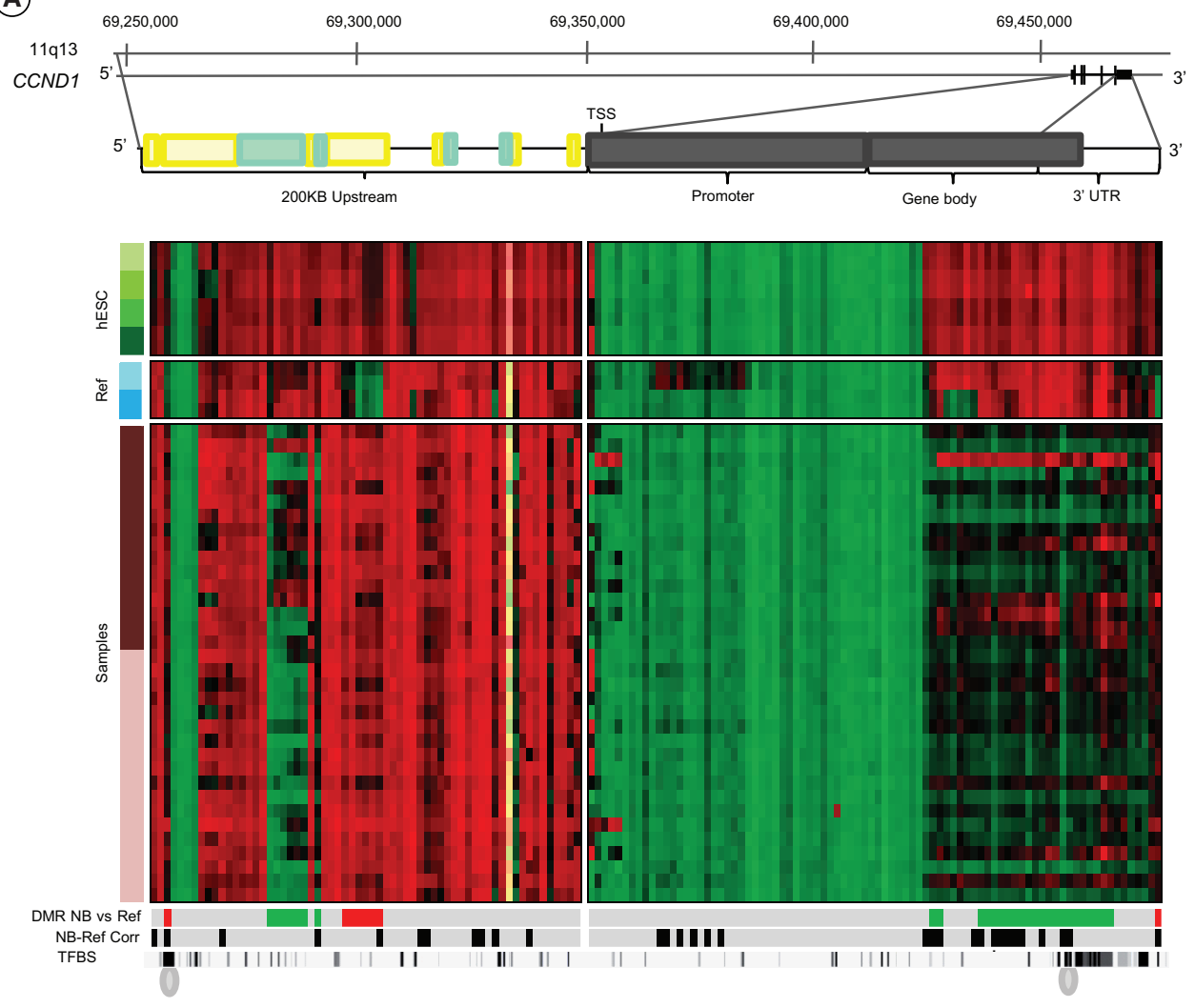

$\beta$-value levels $\mathrm{CpG}$ Min Max non-CpG Min Max

Samples
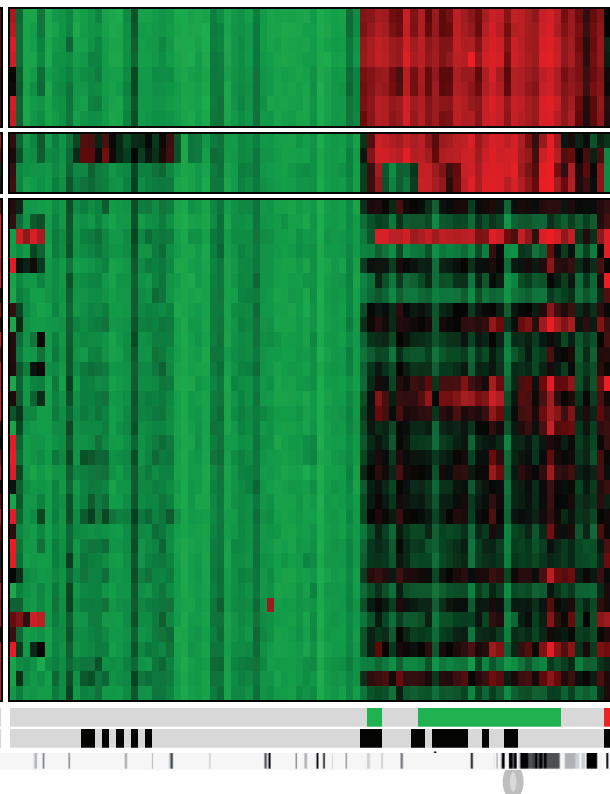

Chromatin states

Enhancer Transcription

Enhancer Transcriptio
(B)

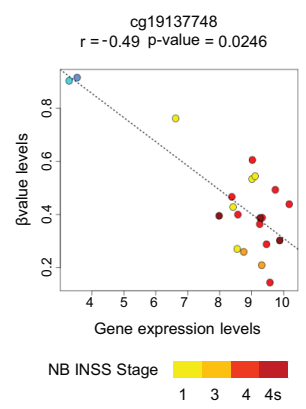

(C)

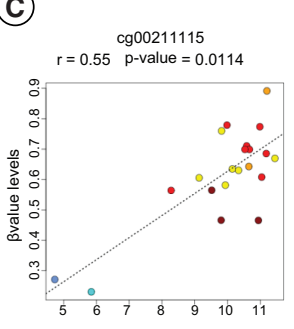

Gene expression levels

(D)

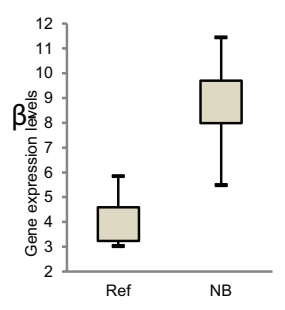

Figure 5. CCND1 gene methylation and expression analysis. (A) DNA methylation landscape of CpGs and non-CpGs associated with the CCND1 gene. From top to bottom, we provide the following information: location and structure of the gene, chromatin and transcriptional domains, heatmap with DNA methylation levels, presence of hypomethylated (green) or hypermethylated (red) regions, presence of significant correlation between DNA methylation and gene expression levels (black) and TFBs density [37]. (B) Scatterplot with linear trend line of CpG (cg19137748) at CCND1 gene-body representing a negative correlation with gene expression. (C) Scatterplot with linear trend line of CpG (cg00211115) located 200 KB upstream of CCND1 TSS representing a positive correlation with gene expression. (D) Differential gene expression levels between neuroblastoma and reference samples. AG: Adrenal gland; $\beta$-value levels: DNA methylation level (value); FB: Fetal brain; hESC: Human embryonic stem cell; NB INSS Stage: Stage according to the International Neuroblastoma Staging System.

Recent studies have reported that non-CpG methylation is established de novo during neuronal differentiation $[11,45]$. In neuroblastoma, high-risk tumors which are mainly composed by undifferentiated or poorly differentiated neuroblasts, occasionally exhibit an increase in histologic differentiation in response to chemotherapy. We analyzed non-CpG methylation levels by bisulfite pyrosequencing of seven high-risk tumors with available matched pairs of tumor specimens obtained at diagnosis and at conclusion of the induction chemotherapy (Supplementary Table 1). All postchemotherapy tumor samples exhibited an increase (range $4-23 \%$ of methylation increase) of non-CpG methylation levels as compared with the matched diagnosis tumor sample. These methylation changes were associated with a decrease of $A L K$ gene expression and discrete signs of therapy-related differentiation as revealed by the increase of neuronal cell differentiation markers (NEFL and TUBB3) and morphological changes of neuroblastic cells (increased cytoplasm and/or ganglion-like morphology) (Figure 6E \& F; Supplementary Figure 7). Posttreatment tumors with neuroblastic undifferentiated areas interspersed with differentiating foci or ganglionar-like cells showed changes in expression patterns of ALK protein, being immunohistochemical staining lowest in the more differentiated ganglionar cells (Figure 6G \& H). 
Altogether, these observations suggest that non$\mathrm{CpG}$ methylation is modulated during neuroblastoma differentiation and these changes may be association with $A L K$ gene expression changes.

\section{Discussion}

Historically, DNA methylation changes in cancer have been described in CGIs and promoter regions [46]. Recent genome-wide studies are starting to reveal a role for DNA methylation outside such genomic contexts $[12,41,47-48]$.
However, the great majority of studies that have investigated DNA methylation in neuroblastoma were focused significantly on the analysis of $\mathrm{CpG}$ islands and promoter regions of genes with potential prognostic relevance [2029]. Such studies have rarely provided insights into DNA methylation changes outside these well-described sites. Our study shows that in neuroblastoma, a developmental cancer, DNA methylation changes frequently affect intragenic and intergenic regions. These changes in part affect apparently nonfunctional domains such as hetero-
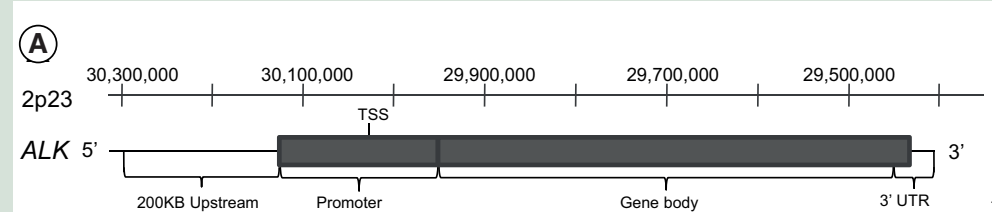

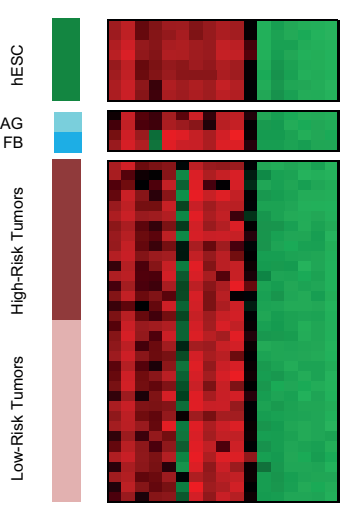

(D)

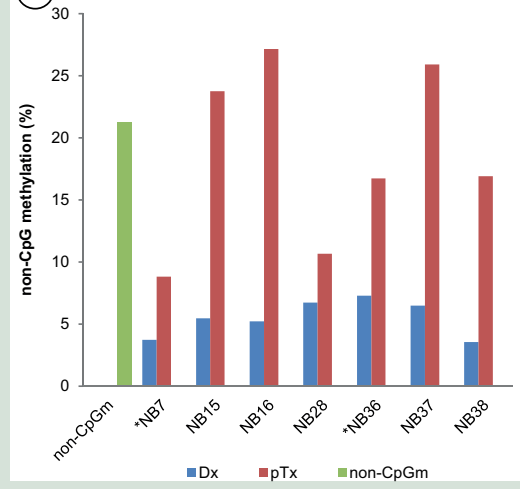

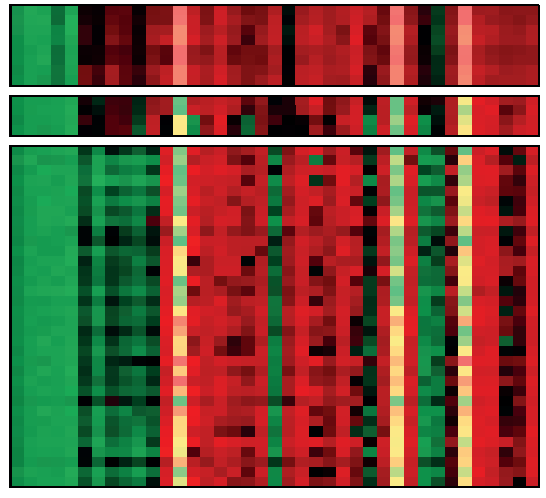

(E)

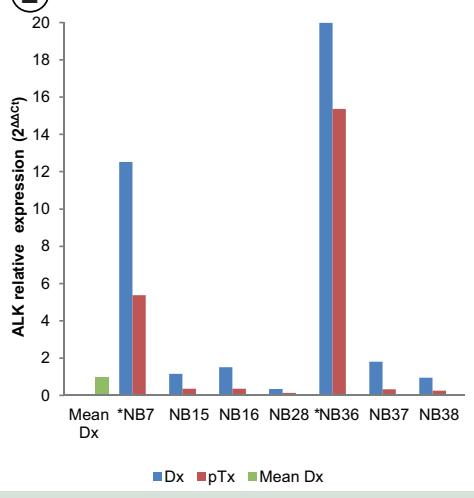

(B)

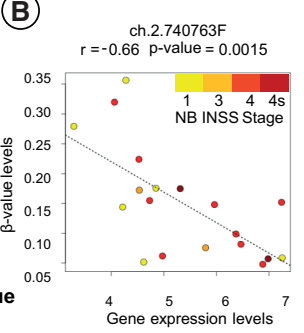

(C)

(D)
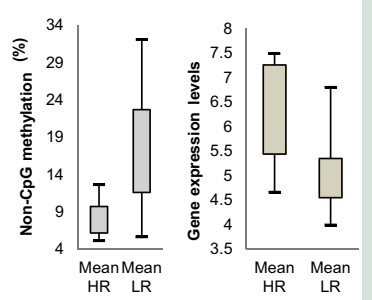

(F)

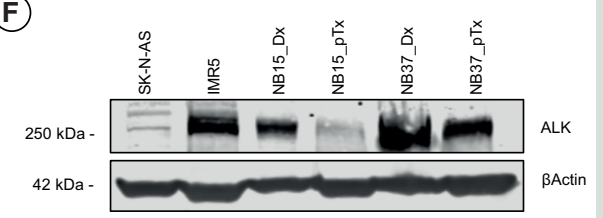

non-CpG
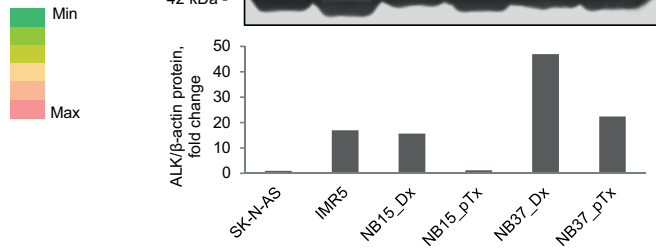

(G)

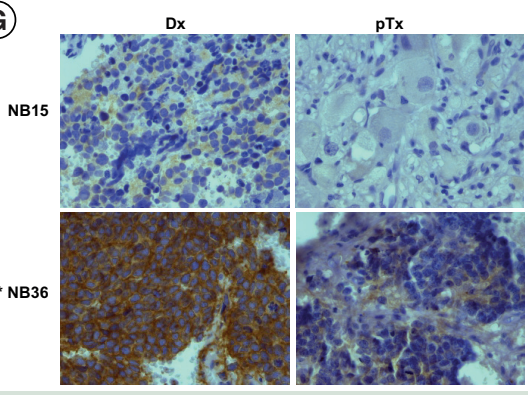

Figure 6. ALK gene methylation and expression analysis. (A) DNA methylation landscape of CpGs and non-CpGs in the $A L K$ gene. From top to bottom: location and structure of the gene and heatmap with DNA methylation levels. (B) Scatterplot with linear trend line of non-CpG (ch.2.740763F) at $A L K$ gene-body representing a negative correlation with gene expression. (C \& D) ALK non-CpG methylation (C) and expression (D) differences between high- and low-risk neuroblastomas. (E) ALK non-CpG methylation levels at the moment of diagnosis and following chemotherapy. non-CpGm: reference levels of methylated tumors. (F) $A L K$ therapy-induced gene expression changes. Mean Dx: mean expression levels of ALK non-amplified neuroblastomas. (G) Protein changes in two exemplary cases at diagnosis and following chemotherapy as compared with neuroblastoma cell lines with low (SK-N-AS) and high ALK protein levels (IMR5). (H) Inmunohistochemical staining of $A L K$ amplified and nonamplified neuroblastoma tumors at diagnosis and after chemotherapy. Neuroblastoma cells with morphological differentiation features show lowest protein staining levels. $\beta$-value levels: DNA methylation level (value); Dx: Sample obtained at diagnosis; hESC: Human embryonic stem cell; HR: Highrisk neuroblastoma; LR: Low-risk neuroblastoma; NB INSS Stage: Stage according to the International Neuroblastoma Staging System; pTx: Sample obtained at conclusion of chemotherapy; Ref: Reference samples: Adrenal gland (AG) and fetal brain (FB); (*): ALK amplified neuroblastoma. 
chomatin and in part regions involved in gene regulation such as enhancers, further supporting an important role for nonpromoter methylation $[18,48]$. However, poised promoters or polycomb repressed regions were also found to be recurrently affected by methylation changes, suggesting thus a tight control of developmental gene expression and subsequently of differentiation pathways in neuroblastoma.

One of the most relevant findings of our study is the presence of DNA methylation at non-CpG sites in neuroblastoma. Recent studies have reported the presence of non-CpG methylation in human embryonic stem cells (hESCs) and in ectoderm-derived tissues (cerebellum, brain cortex and olfactory bulb), the latter suggesting a cell-type specific epigenetic mark [11,13-15,45]. In these studies, non-CpG methylation levels were found to be negligible in fetal cortex, suggesting that the non-CpG methylation detected in ESCs is largely lost upon cell differentiation and that reconfiguration occurs during fetal to young adult neuron development $[14,49]$. In neurons, non-CpG methylation is well conserved and accumulates to become the main form of methylation in the human neuron genome $[11,14,49]$. However, the biological importance of this epigenetic phenomenon is still unclear and a matter of discussion.

Consistent with previous reports, we observed extensive non-CpG methylation in hESCs and very low levels in the fetal brain samples. In neuroblastoma, we observed non-CpG methylation mostly in the clinically more favorable neuroblastoma tumors, where it was greatly associated with genes related to cell differentiation and embryonic development. Interestingly, among genes targeted by non-CpG methylation we identified the $A L K$ gene. $A L K$ is predominantly expressed in the developing nervous system and its expression declines after birth [50]. Recently, $A L K$ has been identified as a gene upregulated in unfavorable neuroblastoma, suggesting an oncogenic role for $A L K$ in neuroblastoma pathogenesis [6,32,50-53]. Although $A L K$ point mutations are one of the most critical alterations in hereditary neuroblastoma, activating mutations and gene amplification have been reported for a limited number of sporadic neuroblastoma tumors (3-11\% and 3-4\%, respectively) [6,52,54]. Hence, the mechanism whereby $A L K$ is upregulated in clinically unfavorable neuroblastomas has not been completely elucidated. We found $A L K$ non-CpG methylation to be associated with clinically favorable tumors and low expression levels, but absent in clinically unfavorable. Interestingly, high-risk tumors restored non-CpG methylation following chemotherapy. Therapy-induced neural differentiation has been described in high-risk, poorly differentiated, neuroblastoma tumors and is characterized by rosette formation, ganglionar cells and the formation of neuropil. We observed how non-CpG methylation in post-treatment high-risk tumors was associated with a decrease in $A L K$ expression and discrete therapy-induced differentiation, as revealed by the morphological changes and the expression of neuronal cell-specific differentiation markers. Thus, the detected increase of cytosine methylation outside CpG dinucleotides provides support for a potential role of this methylation event in the differentiation process of neuroblastic cells and the transcriptional regulation of developmental genes such as $A L K$. These associations require further investigation to establish the precise role of non-CpG methylation in the pathogenesis of neuroblastoma.

These results also reveal DNA methylation changes potentially induced by chemotherapy. This raises the point, however, that diagnostic and postchemotherapy neuroblastoma may be epigenetically different. Moreover, postchemotherapy DNA methylation changes may target genes with recurrent genetic alterations in neuroblastoma, and may thus affect the same genes and pathways. These findings highlight an important aspect associated with the clinical evolution of high-risk neuroblastoma and warrant a genome-wide analysis of epigenetic changes that may affect the DNA methylome of this pediatric tumor as a result of chemotherapy.

Previous reports have suggested that genetic and epigenetic alterations may target similar genes and pathways in cancer $[42,55]$. We observed DNA methylation changes in a significant portion $(p<0.005)$ of genes previously described mutated or with recurrent genetic alterations in neuroblastoma [4-7,24]. Overall, these genes showed a preponderant $\mathrm{CpG}$ loss of methylation that greatly targeted gene-body regions outside CGIs. CCND1 was among genes with extensive $\mathrm{CpG}$ methylation changes, which targeted gene-body and 3'-UTR regions of the gene. Notably, CCND1 promoter region was devoid of DNA methylation both in neuroblastoma as in all reference tissues. These results confirm and extend our previous findings, and suggest that overexpression of CCND1 in neuroblastoma tumors is not associated with methylation abnormalities of the promoter region [38]. CCND1 is highly expressed in various types of solid tumors and in almost all neuroblastoma tumors [56]. The cause of CCND1 overexpression is greatly unknown. We identified expression-related methylation sites at $\mathrm{CpGs}$ located upstream $(160-190 \mathrm{~kb})$ to the TSS of the CCND1 gene, which were at regions enriched for potential regulatory elements (i.e., enhancers). At these distal regulatory elements, we observed enrichment for bindings sites of TFs recently reported to be involved in the transcriptional regulation of CCND1 such as BHLHE40 (DEC-1) and GATA3 (negative and positive regulation, respectively) [56,57]. Recent studies in breast cancer provide experimental evidence for the presence in this distal region of functional enhancers of the CCND1 gene. 
Moreover, these studies revealed a relationship between DNA methylation variations of these enhancers and CCND1 expression $[44,58]$. We can thus speculate a similar mechanistic link between DNA methylation levels of distal upstream regions enriched for enhancers and CCND1 expression in neuroblastoma.

CpG aberrant methylation has been considered a promising predictive marker in cancer. The simultaneous methylation of multiple CGIs, CpG island methylator phenotype (CIMP), has showed a strong predictive power in many cancers, including in neuroblastoma [2123,59]. Consistent with these studies, our findings confirm a high extent of CGI methylation targeting CGIs at promoter regions in clinically unfavorable neuroblastoma tumors. In neuroblastoma, the CIMP is exemplified by the methylation state of the $P C D H B$ gene cluster [21-23,59]. Consistent with previous reports, high-risk tumors in our cohort showed higher, though in most cases not statistically significant, $\mathrm{CpG}$ methylation levels at $\mathrm{CpG}$ islands of the genes [21-23,59]. However, we observed a significant $(\mathrm{p}<0.05)$ association between methylation changes at nonpromoter $\mathrm{CpG}$ sites located outside CGIs of the PCDHB7 gene and overall survival of neuroblastoma patients included in our study. Our study revealed extensive, genome-wide nonpromoter methylation changes at CpG CGIs and non-CpG sites both inside and outside CGIs and gene-body regions significantly associated with neuroblastoma clinical behavior. These findings support the rational for further evaluation of these epigenetic changes as a novel source for novel epigenetic markers for this developmental tumor. It is worth mentioning that non-CpG methylation is asymmetrical, thus methylation variations affecting non-CpGs are small, sometimes subtle. For this reason, the identified differentially methylated CpG sites could represent a more robust source of new epigenetic biomarkers for neuroblastoma. However, our conclusions are based on a small cohort of patients and therefore warrant further investigation and validation in larger independent cohorts of neuroblastoma patients.

\section{Conclusion}

This is, to the best of our knowledge, the first DNA methylation study in neuroblastoma that uses highdensity microarrays to define the epigenetic landscape of this pediatric tumor and its potential clinicopathological impact. Our results reveal that nonpromoter methylation at both $\mathrm{CpG}$ and non-CpG sites may have functional and clinical implications in the pathogenesis of this pediatric tumor. Interestingly, the presence of non-CpG methylation has not been previously described in neuroblastoma and seems to be associated with the differentiation status of the neoplastic cells and the expression of some key genes such as $A L K$. This study provides new insights into the pathogenesis and clinical behavior of this pediatric tumor and provides a rationale for further evaluation of the identified epigenetic modifications as a source for novel biomarkers and therapeutic targets for this, and potentially, other developmental tumors.

\section{Data deposition}

Methylation and expression microarray data have been deposited at NCBI Gene Expression Omnibus data repository (GEO [60]) with Accession number GSE54719 and GSE54720, respectively; on Superseries record GSE54721.

\section{Links to deposited data}

Superseries record [61], methylation microarray data [62] expression microarray data [63].

\section{Acknowledgements}

Authors acknowledge G Garcia-Castellví for fundraising and the "Biobanc de I'Hospital Infantil Sant Joan de Déu per a la Investigació" integrated in the National Network Biobanks of ISCIII for tissue samples. Authors thank LC Laurent for her assistance in obtaining methylation data from hESCs, J Ríos for statistical advice and S Sánchez Molina for precious technical support.

\section{Financial \& competing interests disclosure}

This work was supported by the NEN association (Association of Families and Friends of Patients with Neuroblastoma) (to S Gómez), Hospital Sant Joan de Déu, Barcelona, Spain (BR201102 to G Mayol) and Spanish Government (Instituto de Salud Carlos III grant to C Lavarino and Ramon y Cajal Grant to JI Martín-Subero). The authors have no other relevant affiliations or financial involvement with any organization or entity with a financial interest in or financial conflict with the subject matter or materials discussed in the manuscript apart from those disclosed.

No writing assistance was utilized in the production of this manuscript.

\section{Ethical conduct of research}

The authors state that they have obtained appropriate institutional review board approval or have followed the principles outlined in the Declaration of Helsinki for all human or animal experimental investigations. In addition, for investigations involving human subjects, informed consent has been obtained from the participants involved..

\section{Open access}

This work is licensed under the Creative Commons Attribution-NonCommercial 4.0 Unported License. To view a copy of this license, visit http://creativecommons. org/licenses/by-nc-nd/4.0/ 
Executive summary

- This is the first comprehensive DNA methylation study in neuroblastoma using high-density microarrays.

- Our findings revealed that DNA methylation in neuroblastoma affects not only promoters but also intragenic and intergenic regions at both $\mathrm{CpG}$ and non-CpG sites.

- Nonpromoter methylation changes targeted functional chromatin domains of development and cancerrelated genes.

- Tumors with diverse clinical-risk showed differences affecting CpG and, remarkably, non-CpG sites.

- These epigenetic changes may have functional and clinical implications in the pathogenesis of this pediatric tumor.

- Non-CpG methylation is present in clinically favorable neuroblastoma tumors.

- Non-CpG methylation seems to be associated with the differentiation status of the neoplastic cells and the expression of some key genes such as $A L K$.

- High-risk tumors increased methylation at non-CpGs and decreased $A L K$ expression following chemotherapy.

- This study provides a source for novel epigenetic markers and therapeutic targets for this, and potentially, other developmental tumors.

\section{References}

Papers of special note have been highlighted as:

- of interest; $\bullet \bullet$ of considerable interest

1 Maris JM, Hogarty MD, Bagatell R, Cohn SL. Neuroblastoma. Lancet 369(9579), 2106-2120 (2007).

- Review of the clinical characteristics and molecular pathogenesis of neuroblastoma.

2 Brodeur GM. Neuroblastoma: biological insights into a clinical enigma. Nat. Rev. Cancer. 3(3), 203-216 (2003).

- Review of the clinical characteristics and molecular pathogenesis of neuroblastoma.

3 Cohn SL, Pearson AD, London WB et al. The International Neuroblastoma Risk Group (INRG) classification system: an INRG Task Force report. J. Clin. Oncol. 27(2), 289-297 (2009).

4 Cheung NK, Zhang J, Lu C et al. Association of age at diagnosis and genetic mutations in patients with neuroblastoma. JAMA 307(10), 1062-1071 (2012).

5 Molenaar JJ, Koster J, Zwijnenburg DA et al. Sequencing of neuroblastoma identifies chromothripsis and defects in neuritogenesis genes. Nature 483(7391), 589-593 (2012).

6 Pugh TJ, Morozova O, Attiyeh EF et al. The genetic landscape of high-risk neuroblastoma. Nat. Genet. 45(3), 279-284 (2013).

7 Sausen M, Leary RJ, Jones S et al. Integrated genomic analyses identify ARID1A and ARID1B alterations in the childhood cancer neuroblastoma. Nat. Genet. 45(1), 12-17 (2013).

8 Smith ZD, Meissner A. DNA methylation: roles in mammalian development. Nat. Rev. Genet. 14(3), 204-220 (2013).

9 Baylin SB, Jones PA. A decade of exploring the cancer epigenome - biological and translational implications. Nat. Rev. Cancer. 11(10), 726-734 (2011).

10 Dyachenko OV, Schevchuk TV, Kretzner L, Buryanov YI, Smith SS. Human non-CG methylation: are human stem cells plant-like? Epigenetics 5(7), 569-572 (2010).

11 Lister R, Mukamel EA, Nery JR et al. Global epigenomic reconfiguration during mammalian brain development. Science 341(6146), 1237905 (2013).
-• Genome-wide analysis of non-CpG methylation during human brain developmental stages.

12 Lister R, Pelizzola M, Dowen RH et al. Human DNA methylomes at base resolution show widespread epigenomic differences. Nature 462(7271), 315-322 (2009).

-. Non-CpG methylation modifications induced upon differentiation of embryonic stem cells.

13 Lister R, Pelizzola M, Kida YS et al. Hotspots of aberrant epigenomic reprogramming in human induced pluripotent stem cells. Nature 471(7336), 68-73 (2011).

14 Ramsahoye BH, Biniszkiewicz D, Lyko F, Clark V, Bird AP, Jaenisch R. Non-CpG methylation is prevalent in embryonic stem cells and may be mediated by DNA methyltransferase 3a. Proc. Natl Acad. Sci. USA 97(10), 5237-5242 (2000).

15 Hon GC, Rajagopal N, Shen Y et al. Epigenetic memory at embryonic enhancers identified in DNA methylation maps from adult mouse tissues. Nat. Genet. 45(10), 1198-1206 (2013).

16 Kreck B, Richter J, Ammerpohl O et al. Base-pair resolution DNA methylome of the EBV-positive Endemic Burkitt lymphoma cell line DAUDI determined by SOLiD bisulfitesequencing. Leukemia 27(8), 1751-1753 (2013).

17 Berman BP, Weisenberger DJ, Aman JF et al. Regions of focal DNA hypermethylation and long-range hypomethylation in colorectal cancer coincide with nuclear lamina-associated domains. Nat. Genet. 44(1), 40-46 (2012).

18 Kulis M, Queiros AC, Beekman R, Martin-Subero JI. Intragenic DNA methylation in transcriptional regulation, normal differentiation and cancer. Biochim. Biophys. Acta 1829(11), 1161-1174 (2013).

19 Truong M, Yang B, Wagner J, Desotelle J, Jarrard DF. Analysis of promoter non-CG methylation in prostate cancer. Epigenomics 5(1), 65-71 (2013).

20 Yang Q, Kiernan CM, Tian Y et al. Methylation of CASP8, DCR2, and HIN-1 in neuroblastoma is associated with poor outcome. Clin. Cancer. Res. 13(11), 3191-3197 (2007).

21 Abe M, Westermann F, Nakagawara A, Takato T, Schwab $\mathrm{M}$, Ushijima T. Marked and independent prognostic 
significance of the $\mathrm{CpG}$ island methylator phenotype in neuroblastomas. Cancer. Lett. 247(2), 253-258 (2007).

22 Banelli B, Brigati C, Di Vinci A et al. A pyrosequencing assay for the quantitative methylation analysis of the PCDHB gene cluster, the major factor in neuroblastoma methylator phenotype. Lab. Invest. 92(3), 458-465 (2012).

23 Asada K, Watanabe N, Nakamura Y et al. Stronger prognostic power of the $\mathrm{CpG}$ island methylator phenotype than methylation of individual genes in neuroblastomas. Japanese J. Clin. Oncol. 43(6), 641-645 (2013).

- The $\mathrm{CpG}$ island methylator phenotype prognostic power in neuroblastoma.

24 Koyama H, Zhuang T, Light JE et al. Mechanisms of CHD5 inactivation in neuroblastomas. Clin. Cancer. Res. 18(6), 1588-1597 (2012).

25 Hoebeeck J, Michels E, Pattyn F et al. Aberrant methylation of candidate tumor suppressor genes in neuroblastoma. Cancer. Lett. 273(2), 336-346 (2009).

26 Haruta M, Kamijo T, Nakagawara A, Kaneko Y. RASSF1A methylation may have two biological roles in neuroblastoma tumorigenesis depending on the ploidy status and age of patients. Cancer. Lett. 348(1-2), 167-176 (2014).

27 Teitz T, Wei T, Valentine MB et al. Caspase 8 is deleted or silenced preferentially in childhood neuroblastomas with amplification of MYCN. Nat. Med. 6(5), 529-535 (2000).

28 Yanez Y, Grau E, Rodriguez-Cortez VC et al. Two independent epigenetic biomarkers predict survival in neuroblastoma. Clin. Epigenetics 7(1), 16 (2015).

29 Dreidax D, Bannert S, Henrich KO et al. p19-INK4d inhibits neuroblastoma cell growth, induces differentiation and is hypermethylated and downregulated in MYCNamplified neuroblastomas. Hum. Mol. Genet. 23(25), 6826-6837 (2014).

30 Brodeur GM, Pritchard J, Berthold F et al. Revisions of the international criteria for neuroblastoma diagnosis, staging, and response to treatment. J. Clin. Oncol. 11(8), 1466-1477 (1993).

31 Lavarino C, Garcia I, Mackintosh C et al. Differential expression of genes mapping to recurrently abnormal chromosomal regions characterize neuroblastic tumours with distinct ploidy status. BMC Med. Genomics 1, 36 (2008).

32 Bagci O, Tumer S, Olgun N, Altungoz O. Copy number status and mutation analyses of anaplastic lymphoma kinase (ALK) gene in 90 sporadic neuroblastoma tumors. Cancer Lett. 317(1), 72-77 (2012).

33 Garcia I, Mayol G, Rios J et al. A three-gene expression signature model for risk stratification of patients with neuroblastoma. Clin. Cancer Res. 18(7), 2012-2023 (2012).

34 Bibikova M, Barnes B, Tsan C et al. High density DNA methylation array with single $\mathrm{CpG}$ site resolution. Genomics 98(4), 288-295 (2011).

35 Nazor KL, Altun G, Lynch C et al. Recurrent variations in DNA methylation in human pluripotent stem cells and their differentiated derivatives. Cell Stem Cell 10(5), 620-634 (2012).

36 Kent WJ, Sugnet CW, Furey TS et al. The human genome browser at UCSC. Genome Res. 12(6), 996-1006 (2002).
37 Roadmap Epigenomics C, Kundaje A, Meuleman W et al. Integrative analysis of 111 reference human epigenomes. Nature 518(7539), 317-330 (2015).

-. Analysis of regulatory elements in genomes of human tissues and cells - embryonic and adult, diseased and healthy.

38 Mayol G, Martin-Subero JI, Rios J et al. DNA hypomethylation affects cancer-related biological functions and genes relevant in neuroblastoma pathogenesis. PLoS ONE 7(11), e48401 (2012).

39 Gautier L, Cope L, Bolstad BM, Irizarry RA. affyanalysis of Affymetrix GeneChip data at the probe level. Bioinformatics 20(3), 307-315 (2004).

40 Garcia I, Mayol G, Rodriguez E et al. Expression of the neuron-specific protein CHD5 is an independent marker of outcome in neuroblastoma. Mol. Cancer 9, 277 (2010).

41 Kulis M, Heath S, Bibikova M et al. Epigenomic analysis detects widespread gene-body DNA hypomethylation in chronic lymphocytic leukemia. Nat. Genet. 44(11), 1236-1242 (2012).

42 Chan TA, Glockner S, Yi JM et al. Convergence of mutation and epigenetic alterations identifies common genes in cancer that predict for poor prognosis. PLoS. Med. 5(5), e114 (2008).

43 Okawa ER, Gotoh T, Manne J et al. Expression and sequence analysis of candidates for the $1 \mathrm{p} 36.31$ tumor suppressor gene deleted in neuroblastomas. Oncogene 27(6), 803-810 (2008).

44 Aran D, Hellman A. DNA methylation of transcriptional enhancers and cancer predisposition. Cell 154(1), 11-13 (2013).

-. Expression-correlated methylation in distal sites in the CCND1 breast cancer risk locus.

45 Guo JU, Su Y, Shin JH et al. Distribution, recognition and regulation of non-CpG methylation in the adult mammalian brain. Nat. Neurosci. 17(2), 215-222 (2014).

-. Provides insights for understanding the role of non- $\mathrm{CpG}$ methylation changes during neuronal maturation.

46 Esteller M. Epigenetics in cancer. N. Engl. J. Med. 358(11), 1148-1159 (2008).

47 Ball MP, Li JB, Gao Y et al. Targeted and genome-scale strategies reveal gene-body methylation signatures in human cells. Nat. Biotechnol. 27(4), 361-368 (2009).

48 Jones PA. Functions of DNA methylation: islands, start sites, gene bodies and beyond. Nat. Rev. Genet. 13(7), 484-492 (2012).

49 Kozlenkov A, Roussos P, Timashpolsky A et al. Differences in DNA methylation between human neuronal and glial cells are concentrated in enhancers and non-CpG sites. Nucleic Acids Res. 42(1), 109-127 (2014).

50 Mosse YP, Laudenslager M, Longo L et al. Identification of ALK as a major familial neuroblastoma predisposition gene. Nature 455(7215), 930-935 (2008).

51 Caren H, Abel F, Kogner P, Martinsson T. High incidence of DNA mutations and gene amplifications of the ALK gene in advanced sporadic neuroblastoma tumours. Biochem. J. 416(2), 153-159 (2008). 
52 George RE, Sanda T, Hanna M et al. Activating mutations in ALK provide a therapeutic target in neuroblastoma. Nature 455(7215), 975-978 (2008).

53 Carpenter EL, Mosse YP. Targeting ALK in neuroblastoma - preclinical and clinical advancements. Nat. Rev. Clin. Oncol. 9(7), 391-399 (2012).

- Biology of ALK in the development of neuroblastoma, preclinical and clinical progress and challenges associated with resitance to ALK inhibitors.

54 Passoni L, Longo L, Collini P et al. Mutation-independent anaplastic lymphoma kinase overexpression in poor prognosis neuroblastoma patients. Cancer. Res. 69(18), 7338-7346 (2009).

55 Schuebel KE, Chen W, Cope L et al. Comparing the DNA hypermethylome with gene mutations in human colorectal cancer. PLoS Genet. 3(9), 1709-1723 (2007). Molenaar JJ, Ebus ME, Koster J et al. Cyclin D1 is a direct transcriptional target of GATA3 in neuroblastoma tumor cells. Oncogene 29(18), 2739-2745 (2010).

57 Wang Y, Rao VK, Kok WK et al. SUMO modification of Stra13 is required for repression of cyclin D1 expression and cellular growth arrest. PLoS ONE 7(8), e43137 (2012).
58 French JD, Ghoussaini M, Edwards SL et al. Functional variants at the $11 \mathrm{q} 13$ risk locus for breast cancer regulate cyclin D1 expression through long-range enhancers. Am. J. Hum. Genet. 92(4), 489-503 (2013).

59 Suzuki H, Yamamoto E, Maruyama R, Niinuma T, Kai M. Biological significance of the CpG island methylator phenotype. Biochem. Biophys. Res. Commun. 455(1-2), 35-42 (2014).

60 GEO Expression Omnibus. http://www.ncbi.nlm.nih.gov/geo/

61 Lavarino C. DNA methylation changes at CpG and non$\mathrm{CpG}$ sites are associated with development and clinical behavior in neuroblastoma. www.ncbi.nlm.nih.gov/geo/query/acc.cgi?token

62 Lavarino C. DNA methylation changes at CpG and non$\mathrm{CpG}$ sites are associated with development and clinical behavior in neuroblastoma [methylation]. http://www.ncbi.nlm.nih.gov/geo/query

63 Lavarino C. DNA methylation changes at CpG and nonCpG sites are associated with development and clinical behavior in neuroblastoma [gene expression]. http://www.ncbi.nlm.nih.gov/geo/query 